\title{
Vehicular trajectory estimation utilizing slip angle based on GNSS Doppler/IMU
}

\author{
Kanamu Takikawa ${ }^{1 *}\left(\mathbb{D}\right.$, Yoshiki Atsumi $^{1}$, Aoki Takanose ${ }^{1}$ and Junichi Meguro²
}

\begin{abstract}
Accurate vehicular trajectory estimation is important for the recently developed autonomous driving systems. As the accuracy of the vehicular trajectory estimation is reduced with the slippage that occurs during turning, we propose a method in this study to accurately estimate the trajectory of a vehicle, focusing on the slip angle estimation. Although the two-wheel model is used as a general concept slip angle estimation, the accurate estimation of the parameters was difficult using the conventional methods. Therefore, a global navigation satellite system (GNSS) Doppler was used for parameter estimation. In addition, the roll angle was estimated as it occurs during turning and affects the slip angle of the vehicle. Specifically, we verified the improvement in accuracy of the vehicular trajectory estimation using the cost-effective GNSS Doppler/IMU.
\end{abstract}

Keywords: Vehicle trajectory, Two-wheel model, Autonomous driving systems, Multi-GNSS, Urban area

\section{Introduction}

Highly accurate vehicular trajectory estimation has become imminently important for automated vehicles and advanced driver assistance systems that have been developed in the recent years [1]. Accurate trajectory information has been used in various studies such as constraints for position estimation, 3-D mapping for automated vehicles, route planning, and vehicular control [2-7]. Accuracy is required for trajectory estimation of automated vehicles. The required accuracy is a $2 \mathrm{D}$ error of $0.3 \mathrm{~m}$ per $100 \mathrm{~m}$ of trajectory, which corresponds to the thickness of a tire. Moreover, conventional automated vehicles often use GNSS/IMU systems with expensive sensors, such as fiber-optic gyros, to achieve the required accuracy. However, the cost of using these expensive sensors is an issue. Therefore, the method proposed in this study aims to achieve accurate trajectory estimation using inexpensive sensors. In addition, we focus on the normal driving range of vehicles and aim to increase the accuracy

\footnotetext{
*Correspondence: 193432010@ccmailg.meijo-u.ac.jp

${ }^{1}$ Division of Mechatronics Engineering, Graduate School of Science and Technology, Meijo University, Shiogamaguchi 1-501, Tenpaku-ku, Nagoya, Aichi 468-0073, Japan

Full list of author information is available at the end of the article
}

of the vehicle trajectory estimation performance. A difference is known to occur between the directions of the velocity vector and the heading angle (slip angle) when the vehicle turns. Therefore, the slip angle estimation is generally carried out using a two-wheeled model to improve the accuracy of vehicular trajectory estimations [8]. However, the two-wheeled models require one-byone estimation of multiple parameters, which imparts error biases and makes the accurate estimation of vehicular trajectory difficult.

Therefore, in the proposed method, the parameters of the two-wheeled vehicle model are automatically estimated using GNSS Doppler to estimate the trajectory corrected for the slip angle. The major gap between our method and conventional methods is that we do not estimate the parameters one by one, but rather eliminate variable parameters by rearranging the model equations and estimate fixed parameters. In addition, our method does not require the construction of an observer and can be used universally because it utilizes not only conventional vehicle motion sensors such as IMU but also GNSS Doppler, which is information from outside the vehicle. The slip angle estimated by our method allows us to make corrections to the trajectory, thereby realizing highly 
accurate vehicle trajectory estimation in the range of normal operation.

In order to confirm the effectiveness of the slip angle correction by the proposed method, we focused on the accuracy of the trajectory in the road orthogonal direction and evaluated it using data acquired from two different environments ("Evaluation tests" section). In the evaluation, the high-precision GNSS/IMU system POSLV220 was used as the reference value, and the effectiveness of the proposed method was confirmed by comparing the slip angle estimate by the proposed method and the trajectory estimate after slip angle correction with the reference value, respectively.

This paper is organized as follows. "Related prior research" section introduces the previous studies, "Increasing the accuracy of vehicle trajectory by correcting the slip angle using GNSS Doppler" section describes the proposed method, "Evaluation tests" section summarizes the evaluation results, and "Conclusions" section concludes.

\section{Related prior research}

\section{Overview of conventional trajectory estimation}

Generally, conventional vehicle trajectory estimation utilizes IMUs and wheel speedometers [1, 9, 10]. First, the trajectory can be broken into its east and north components, and the trajectory at time $t$ can be expressed using Eqs. (1) and (2) as follows.

$$
\begin{aligned}
& T_{\text {East }}^{t}=T_{\text {East }}^{t-1}+V \cdot \cos (\psi+\beta) \cdot d t \\
& T_{\text {North }}^{t}=T_{\text {North }}^{t-1}+V \cdot \sin (\psi+\beta) \cdot d t
\end{aligned}
$$

$V$ : velocity, $\psi$ : heading angle, $\beta$ : slip angle.

Here, the heading angle $\psi$ is expressed in the following Eq. (3) using the yaw rate $\dot{\psi}$ :

$$
\psi^{t}=\psi^{t-1}+\left(\dot{\psi}^{t}+\delta \dot{\psi}\right) \cdot \frac{1}{\cos (\theta) \cdot \cos (\varphi)} \cdot d t
$$

$\dot{\psi}$ : yaw rate, $\theta$ : pitch angle, $\varphi$ : roll angle.

Equation (3) considers the error in the IMU yaw rate $\dot{\psi}$ with an offset of $\delta \dot{\psi}$. Furthermore, Eq. (4) presents the consideration of including the scale factor of wheel speed and the effect of the longitudinal slope of the road with respect to $V$.

$$
V=S F \cdot V_{\operatorname{can}} \cos (\theta)
$$

$V_{\text {can }}$ : CAN-bus Velocity, SF: Scale Factor Error.

Using Eqs. (1)-(4), the vehicle trajectory can be calculated from the output of the IMU using the wheel speed. Therefore, the elements of trajectory that need to be estimated are $\psi, \beta, \delta \dot{\psi}, S F, \varphi$, and $\theta$. Among them, a highly accurate estimation method has been proposed for $\psi$, $\delta \dot{\psi}, S F$, and $\theta[1,11]$; therefore, the estimation of the roll angle $\varphi$ and slip angle $\beta$ remains unfulfilled. Thus, in this paper, we focus on the accuracy of the vehicle trajectory in the road orthogonal direction, estimate the slip angle, and correct the estimated slip angle to the vehicle trajectory.

\section{Previous studies on slip angle estimation}

The equipment required to directly measure the slip angle of a vehicle is expensive and difficult to install in a vehicle [8, 12-14]. Consequently, estimation is often preferred and performed, where the direct integration method [8] and the linear observer estimation method [15-17] are used as typical methods for conventional estimation of the slip angle. However, the direct integration method poses a problem of accumulation of noise and offset in the sensor detections owing to the inclusion of an integrator, and the error increases with time. On the contrary, in the observer estimation method, the observer constructs the settings for the estimation. There are two main types of observer estimation methods: model-based observers [18] and kinematics-based observers [15]. Among these, the model-based observer tends to deviate from the actual values due to the mismatch between the tire parameters of the real vehicle and those used in the model. For this reason, kinematicsbased observers are often used. A number of algorithms have been proposed to estimate and adapt the slip angle of a vehicle using kinematics-based observers. For example, in $[19,20]$, a method for estimating the cornering stiffness of a tire at high steering frequency is proposed. Among these studies, the $\beta$-less method [21] is considered to have the highest potential for practical application in the field. This method is based on a two-wheel model (Fig. 1). In the two-wheel model, the slip angles of the left and right front tires of a four-wheeled vehicle are assumed to be the same and treated as equivalent to two

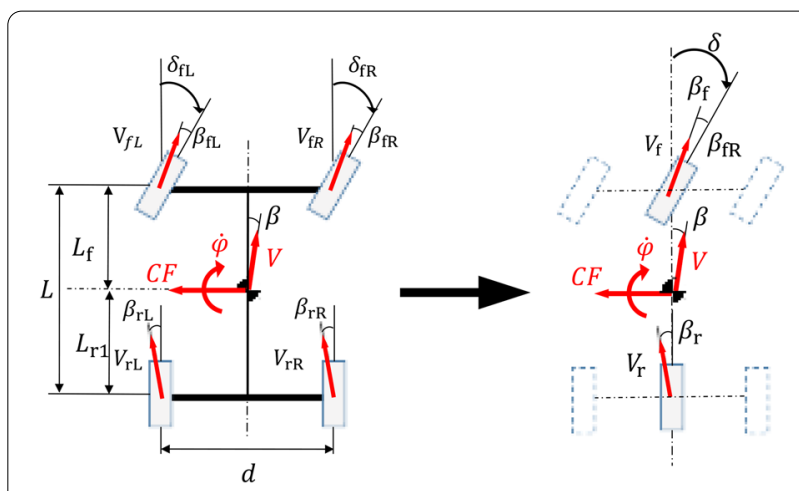

Fig. 1 Two-wheel model 
wheels. The relationship between the slip angle $\beta$ at the center of gravity and the vehicle parameters is shown in Eq. (5).

$$
\beta=\left(\frac{1-\frac{m L_{\mathrm{f}}}{2 L_{\mathrm{f}} K_{\mathrm{r}}} V^{2}}{1-\frac{m}{2 L^{2}}\left(\frac{L_{\mathrm{f}} K_{\mathrm{f}}-L_{\mathrm{r}} K_{\mathrm{r}}}{K_{\mathrm{f}} K_{\mathrm{r}}}\right) V^{2}}\right) \frac{L_{\mathrm{r}} \delta}{L}
$$

$m$ : vehicle weight, $L$ : distance between front and rear wheels, $L_{\mathrm{f}}$ : distance between center of gravity of front wheel, $L_{\mathrm{r}}$ : distance between center of gravity and the rear wheels of the vehicle, $V$ : velocity, $K_{\mathrm{f}}$ : front wheel cornering power, $K_{\mathrm{r}}$ : rear wheel cornering power, $\delta$ : steering angle.

Here, in the previously proposed observer estimation methods, the parameters required for slip angle estimation are measured from the inertial sensor measurements $[12,15]$. However, if these parameters are measured separately, the problem of error bias arises. In particular, the coefficients $K_{\mathrm{f}}$ and $K_{\mathrm{r}}$ are difficult to measure accurately due to various factors such as the effect of tire deformation. There are other limitations such as the need to accurately estimate the steering angle and the need to tune the system for each vehicle [22,23]. In addition, most of the previous studies on vehicle slip angle estimation use Kalman filter as an observer [22-24]. Therefore, in most of the methods, the estimation accuracy of the algorithm is limited to a limited set of operations, subject to experimentation.

\section{Increasing the accuracy of vehicle trajectory by correcting the slip angle using GNSS Doppler Overview of the slip angle estimation}

The proposed method aims to resolve the limitation of determining the parameters of the two-wheeled model for the estimation of the slip angle $\beta$ by the estimating parameters automatically using GNSS Doppler. In addition, the proposed method eliminates the term of steering angle in the two-wheel motorcycle model, and thus does the estimation of steering angle is not required. The proposed method is expected to increase the accuracy of the slip angle estimation to an extent of validating the two-wheel model. Figure 2 shows an overview of the method. Here, in Fig. 2, the heading angle, sensor bias (yaw rate offset), and roll angle are estimated, and then the slip angle is estimated. GNSS systems using dual antennas can measure attitude angles such as heading and roll angles [25-27]. However, these methods can only provide accurate output when there is sufficient signal from the satellite, such as in suburban roads $[25,26]$. On the other hand, when used in urban areas, high accuracy cannot be maintained due to multipath effects. Therefore, it is difficult to estimate the attitude angle using only GNSS systems. Therefore, in order to reduce the effect of multipath and to enable robust

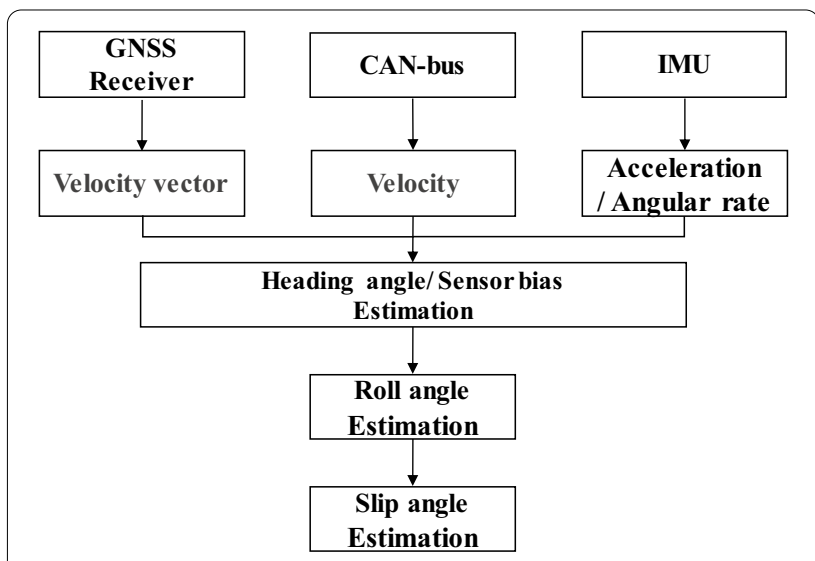

Fig. 2 Overview of the proposal

estimation, an integrated method of GNSS and IMU has been used to estimate the heading angle [1]. Therefore, the slip angle estimation method and the roll angle estimation method in Fig. 2 are proposed in this paper.

In the two-wheel model, the yaw rate $\dot{\psi}$ can be expressed by Eq. (6) [15] as follows.

$$
\dot{\psi}=\left(\frac{1}{1-\frac{m}{2 L^{2}}\left(\frac{L_{f} K_{f}-L_{r} K_{r}}{K_{f} K_{r}}\right) V^{2}}\right) \frac{V \delta}{L}
$$

Therefore, Eqs. (5) and (6) can be combined to obtain the relationship between $\beta$ and $\dot{\psi}$ as given by Eq. (7).

$$
\beta=\dot{\psi}\left(\frac{L_{\mathrm{r}}}{V}-\frac{m L_{\mathrm{f}}}{2 L K_{\mathrm{r}}} V\right)
$$

However, the above equation represents the slip angle $\beta$ at the center of gravity. The slip angle $\beta \_r$ at the center of the rear wheel axle is given by Eq. (8).

$$
\beta_{\mathrm{r}}=\beta-\frac{L_{\mathrm{r}}}{V} \dot{\psi}
$$

Combining Eqs. (7) and (8), we can obtain Eq. (9) as

$$
\beta_{\mathrm{r}}=-\frac{m L_{\mathrm{f}}}{2 L K_{\mathrm{r}}} \dot{\psi} V
$$

As all the parameters in Eq. (9) are fixed and constant parameters, except for $\dot{\psi}$ and $V$, they can be comprehensively represented as the gain $K$ in the following Eq. (10):

$$
\beta_{\mathrm{r}}=-K \dot{\psi} V
$$

where $\dot{\psi} V$ is the product of the yaw rate and velocity and refers to the centrifugal acceleration, so it can be summarized as $G_{y}$ in Eq. (11).

$$
\dot{\psi} V=G_{y}
$$

Therefore, Eq. (11) simplifies to Eq. (12) . 


$$
\beta_{\mathrm{r}}=-K \cdot G_{y}
$$

Furthermore, during motion of the vehicle, the component of gravitational acceleration for the roll angle needs to be considered for Eq. (12) because the component of gravity is included at the time of roll angle generation. Figure 3 shows the relationship between the roll angle and its occurrence. The relationship between the roll angle $\varphi$ and the lateral acceleration $G_{y}$ is expressed in Eq. (13).

$$
G_{y}=\dot{\psi} \cdot V-g \cdot \sin \varphi
$$

Substituting $G_{y}$ from Eq. (13) to Eq. (12), we obtain Eq. (14) as:

$$
\beta_{\mathrm{r}}=-K(\dot{\psi} \cdot V-g \cdot \sin \varphi)
$$

Therefore, the sine component of the roll angle is necessary for the estimation of the slip angle from Eq. (14), which is estimated in the next subsection.

\section{Roll angle estimation}

The roll angle can be estimated with the formula presented by Tseng et al. [28]:

$$
\varphi=\arcsin \left(\frac{V}{g} \cdot \dot{\psi}-\frac{G_{y}}{g}\right)
$$

Moreover, the transverse acceleration $G_{y}$ can be measured from the IMU, and for the error in acceleration of the IMU as $\delta G_{y}^{\text {imu }}$, Eq. (15) can be expressed as Eq. (16).

$$
\varphi=\arcsin \left(\frac{V}{g} \cdot \dot{\psi}-\frac{G_{y}^{\mathrm{imu}}+\delta G_{y}^{\mathrm{imu}}}{g}\right)
$$

Assuming that Eq. (16) holds true, the roll angle $\varphi$ can be estimated upon the correction of $\delta G_{y}^{\text {imu }}$.

Therefore, this study proposes a method for estimating the lateral acceleration error using the relationship between the variation in the heading angle $\psi$ at certain intervals and the integration of the yaw rate $\dot{\psi}$ with the roll angle $\varphi$. The relationship between the variation of

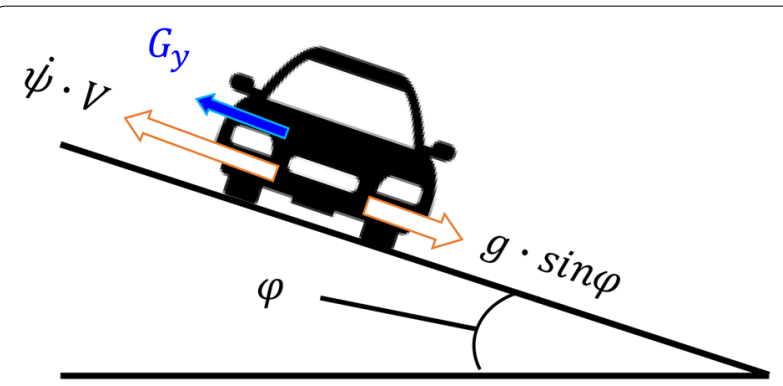

Fig. 3 Effect of road lateral gradient heading angle at certain intervals and the integration of the yaw rate $\dot{\psi}^{2 D}$ as viewed from the horizontal plane can be expressed by Eq. (17).

$$
\psi^{t+n}-\psi^{t}=\int_{t}^{t+n} \dot{\psi}^{2 D} d t
$$

However, the roll angle $\phi$ needs to be considered with respect to the yaw rate in case of a lateral slope on the road surface.

$$
\psi^{t+n}-\psi^{t}=\int_{t}^{t+n} \dot{\psi} \cdot \frac{1}{\cos (\varphi)} d t
$$

Therefore, the expression of roll angle in Eq. (16) can be substituted into the above Eq. (18) to obtain the following Eq. (19).

$$
\begin{aligned}
& \psi^{t+n}-\psi^{t}= \\
& \int_{t}^{t+n} \dot{\psi} \cdot \frac{1}{\cos \left\{\arcsin \left(\frac{V}{g} \cdot \dot{\psi}-\frac{G_{y}^{\mathrm{imu}}+\delta G_{y}^{\mathrm{imu}}}{g}\right)\right\}} d t
\end{aligned}
$$

Equation (19) shows the relationship between the heading angle and the transverse acceleration error at certain intervals. Therefore, the accurate evaluation of the lateral acceleration error through Eq. (19) can be used to accurately estimate the roll angle through Eq. (16).

In this study, we determined $\delta G_{y}^{\text {imu }}$ by utilizing the heading difference between the left and right sides of Eq. (19). By varying the value of $\delta G_{y}^{\text {imu }}$ in Eq. (19), we search for cases where the heading difference is zero or closest to zero, and thus analytically determine $\delta G_{y}^{\mathrm{imu}}$. In Eq. (19), the heading angle $\psi$ can be estimated by using the GNSS Doppler/IMU [1, 29, 30], the velocity $V$ can be measured by the wheel speed sensor, and $G_{y}^{\text {imu }}$ can be measured by the IMU, so only $\delta G_{y}^{\mathrm{imu}}$ remains unknown, for which the estimation can be made. The transverse acceleration error $\delta G_{y}^{\text {imu }}$ cannot be estimated using Eq. (19) when entire right-hand side of the equation becomes zero for $0 \mathrm{rad} / \mathrm{s}$ yaw rate $\dot{\psi}$. Therefore, the computation was performed only at the point where the curve turned once. In this study, we used data for $100 \mathrm{~s}$ with $n=100$. Figure 4 portrays the variation in the heading difference between the left and right sides for different values of $\delta G_{y}^{\text {imu }}$ within -1.5 to $1.5 \mathrm{~m} / \mathrm{s}^{2}$ according to Eq. (19). As shown, the variation of $\delta G_{y}^{\mathrm{imu}}$ produced quadratic curves with inflection points indicating minimum differences between the left and right sides. As each of these inflection points was calculated at a different position on a curve, the average of these values were considered to neutralize the transverse acceleration error $\delta G_{y}^{\mathrm{imu}}$. This estimate did not considerably vary over time so determining sequence was not necessary. Therefore, 


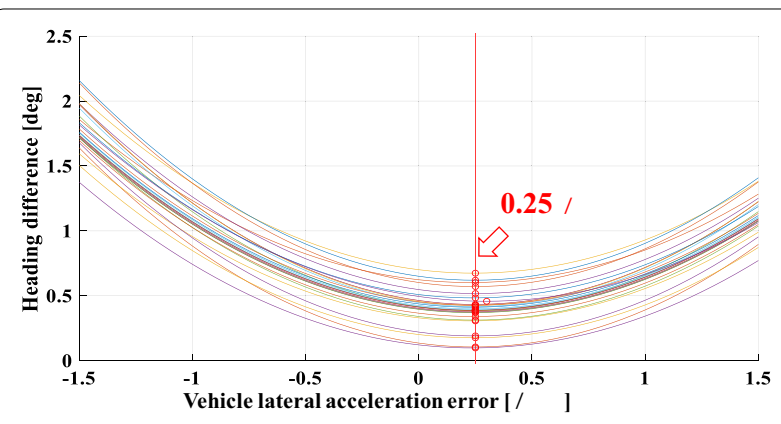

Fig. 4 Estimation results of the lateral acceleration error

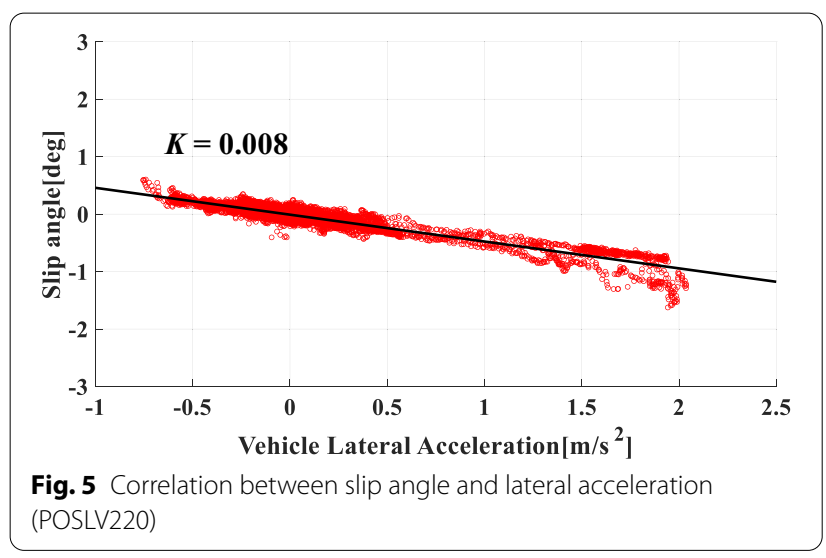

the real-time position estimation can be performed right after conducting the calibration run estimating the initial position. For this dataset, we considered $0.25 \mathrm{~m} / \mathrm{s}^{2}$ as the estimated value.

\section{Parameter estimation for two-wheel model using GNSS Doppler}

Figure 5 presents the relationship between the slip angle and lateral acceleration according to Eq. (14) for a distance of approximately $5 \mathrm{~km}$ around the urban area (Odaiba, Tokyo, Japan). The slip angle and the lateral acceleration were measured by Applanix POSLV220, which is known as a high-precision GNSS/IMU system [31]. The correlation between the slip angles with lateral acceleration can be observed from Fig. 5, where the slope represents the gain $K$ from Eq. (14). Therefore, the slip angle can be estimated by evaluating this relationship.

On the contrary, the velocity vector and heading angle can be estimated by utilizing the GNSS Doppler [32] and combining the IMU with it [1], respectively. In our heading estimation method, we use least-squares fitting of GNSS Doppler and IMU yaw-rate values. Our method is unique in that it uses a long time series of data to determine the multipath. This feature allows us to perform convergence operations while determining and removing outliers, thus improving the heading angle accuracy.

Therefore, this study proposes a method to approximate the variation in heading angles using the GNSS Doppler/IMU and evaluate the first-order approximation of velocity vectors using the GNSS Doppler with the least-squares method, where the slope of the first-order equation is represented by the gain $K$. An overview of the proposed method has been presented in Fig. 6, where $\beta_{r}$ was accumulated according to Eq. (20) by calculating the difference between the directions obtained from the GNSS Doppler/IMU $H_{\text {Doppler/IMU }}$ and the GNSS Doppler $H_{\text {Doppler }}$ at the curve. Moreover, thresholds were set for speed and storage of data beyond a certain speed owing to the higher speed range of the moving object and the higher accuracy of the GNSS Doppler [1, 28, 30, 32].

$$
\beta_{r_{\text {Doppler }}}^{\text {Doppler/IMU }}=H_{\text {Doppler } / \mathrm{IMU}}-H_{\text {Doppler }}
$$

The gain $\mathrm{K}$ was estimated based on the relationship between the slip angle accumulated by Eq. (20) and the lateral acceleration at that time; the gain $\mathrm{K}$ shown in Fig. 7 can be estimated without using a high-precision GNSS/IMU. Here, comparing Figs. 6 and 7, we can see that there is a difference in the estimated value of gain $\mathrm{K}$. The main reason for this is that the POSLV220 can estimate the heading with an accuracy of $0.025^{\circ}$ [31], while

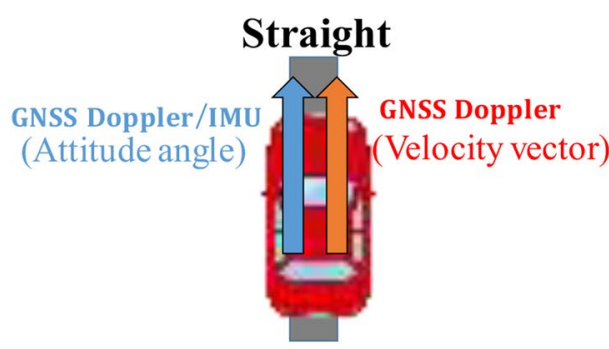

GNSS Doppler/IMU = GNSS Doppler

\section{Curve}

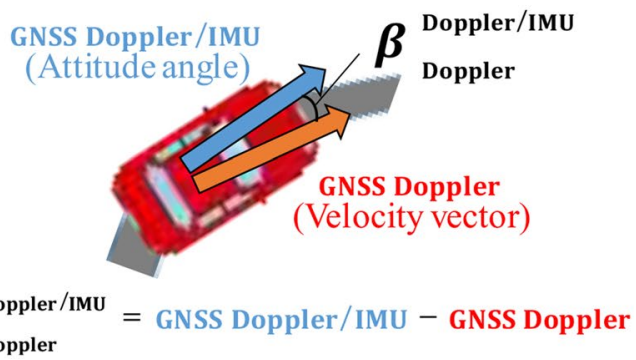

Fig. 6 Overview of the slip angle 


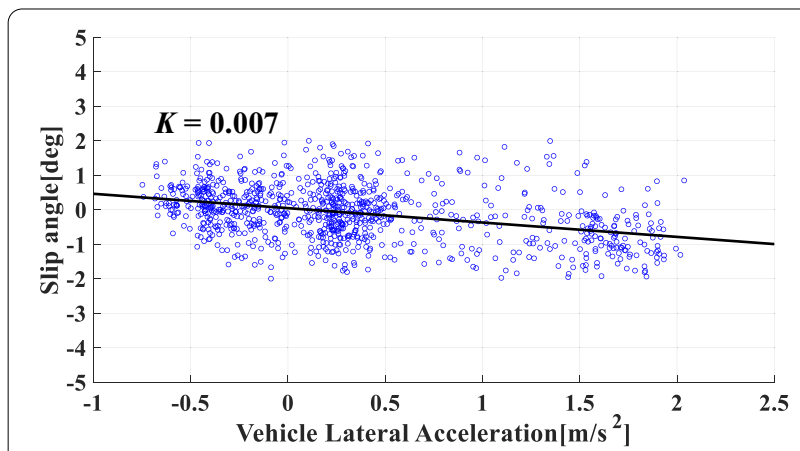

Fig. 7 Relationship between slip angle and lateral acceleration using GNSS/Doppler

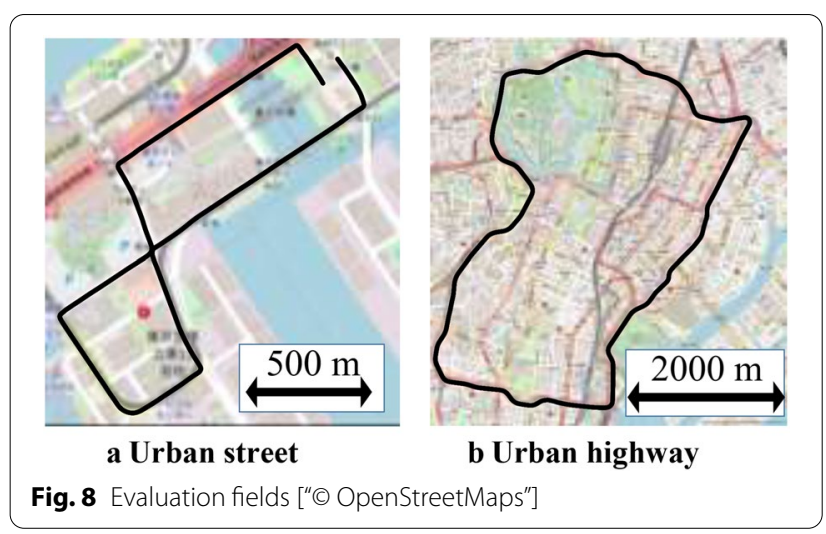

Fig. 8 Evaluation fields ["® OpenStreetMaps"]
GPS + BeiDou + Galileo + QZSS satellite system was used as a GNSS receiver with a reception period of $10 \mathrm{~Hz}$. The MEMS IMU on the TAG264 of Tamagawa Seiki was used with an acquisition period of $50 \mathrm{~Hz}$. An Applanix POSLV220 was used as the reference equipment for an accurate evaluation. Figure 9 shows and Table 1 lists the sensors and POSLV220 that are used in this evaluation. The experimental vehicle used for data collection was a Toyota Alphard. From Table 1, it can be seen that there is a great difference between the sensor cost of the reference and our proposed scheme.

\section{Roll angle estimation results}

The roll angles estimated using the lateral acceleration error $\delta G_{y}$ with our proposed method is shown in Figs. 10 and 11 for the urban street and highway, respectively. In addition, the reference (POSLV220) model measurements along with the raw roll angle estimation (Eq. (16)) data without the correction of $\delta G_{y}$ (No sensor correction) are presented in these figures for comparison. Furthermore, Figs. 12 and 13 show the difference between errors under "No sensor correction" and "Proposal" of the estimated roll angles for the urban street and highway, respectively. Since the IMU and the MEMS-IMU of the POSLV220 are installed at the same location in this experiment, their Roll angle outputs should be consistent. However, since the MEMSIMU is subject to acceleration errors, there will also

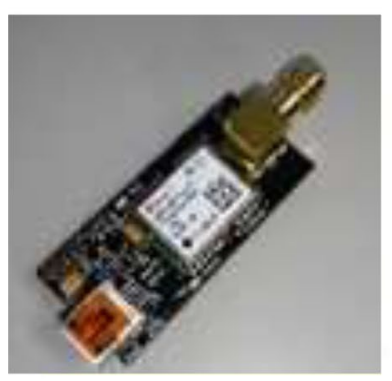

a Ublox M8T

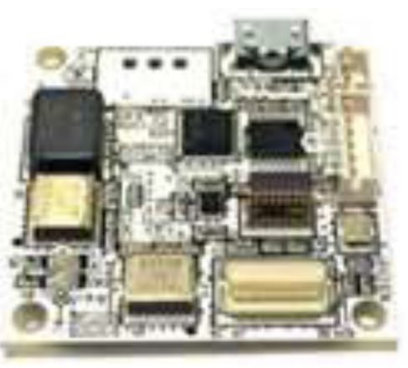

b MEMS IMU

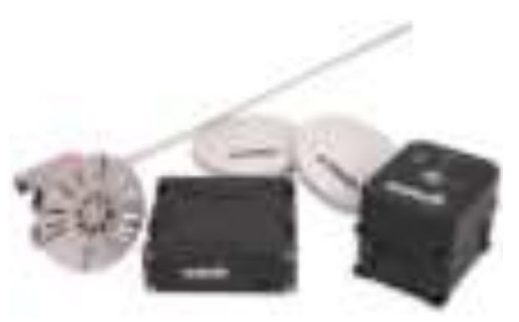

c POSLV220

Fig. 9 Photographs of the sensors and the system

the GNSS Doppler has an error in the estimated heading due to noise [32].

\section{Evaluation tests}

The evaluation tests were conducted on a $5-\mathrm{km}$ course in an urban street (Odaiba, Tokyo, Japan) and a 14-km course on an urban highway (Tokyo Metropolitan Expressway) with a lateral gradient; the courses are shown in Fig. 8. The U-blox M8T with a
Table 1 Equipment used for evaluation

\begin{tabular}{lllc}
\hline & Sensor & Product name & Cost (US\$) \\
\hline Proposal & GNSS Receiver & Ublox M8T(10 Hz) & 100 \\
& GNSS Antenna & Tallysman TW2710 & 100 \\
& MEMS IMU & Tamagawa & 500 \\
& & AU7554 $(50 \mathrm{~Hz})$ & \\
Reference & GNSS Receiver, GNSS & Applanix POSLV220 & 100,000 \\
& Antenna, 3-axis & & \\
& FOG & & \\
\hline
\end{tabular}




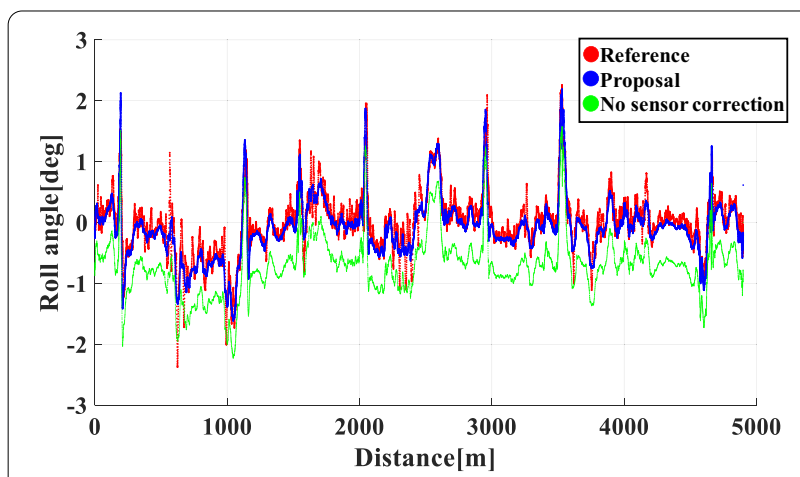

Fig. 10 Estimated roll angle (Urban street)

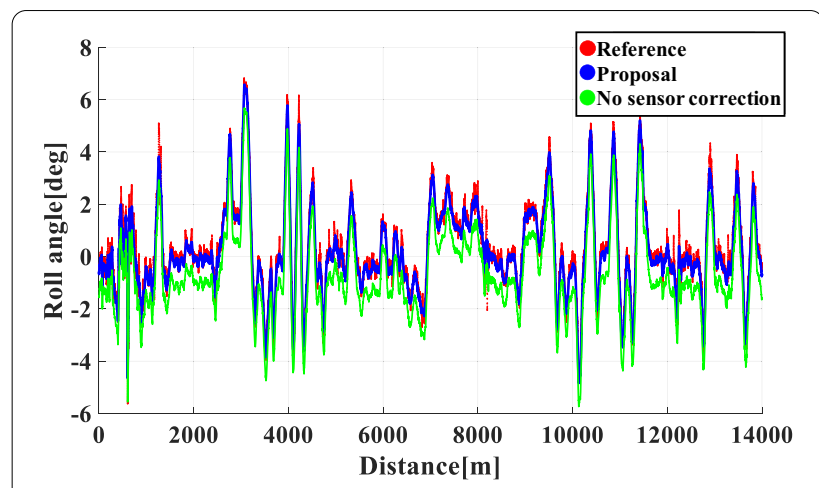

Fig. 11 Estimated roll angle (Urban highway)

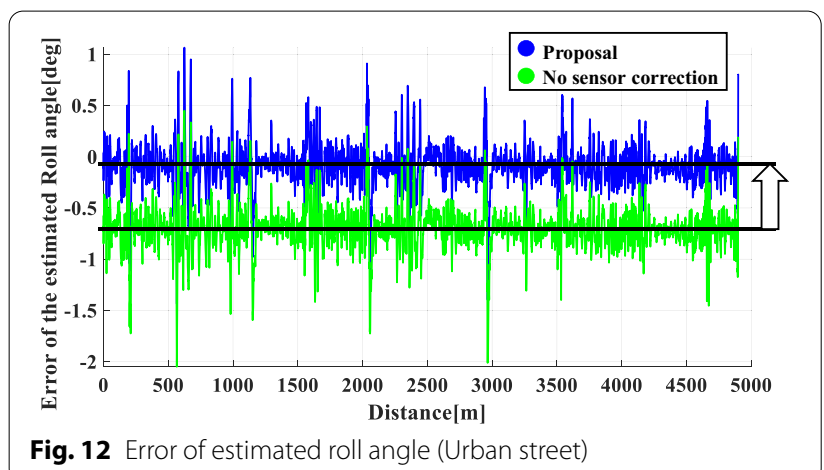

be errors in the estimated roll angle. Here, as shown in Figs. 10-13, the proposed method is able to negate the offset error, indicating that the results are close to the reference. This is due to the correction of the acceleration error estimated by the proposed method. If we look at Figs. 12 and 13, we can see that the estimated roll angles have shifted values. This is because the variation of the acceleration error of the MEMS-IMU used in this experiment is small, and the error of the estimated Roll angle also appears to be constant. Tables 2

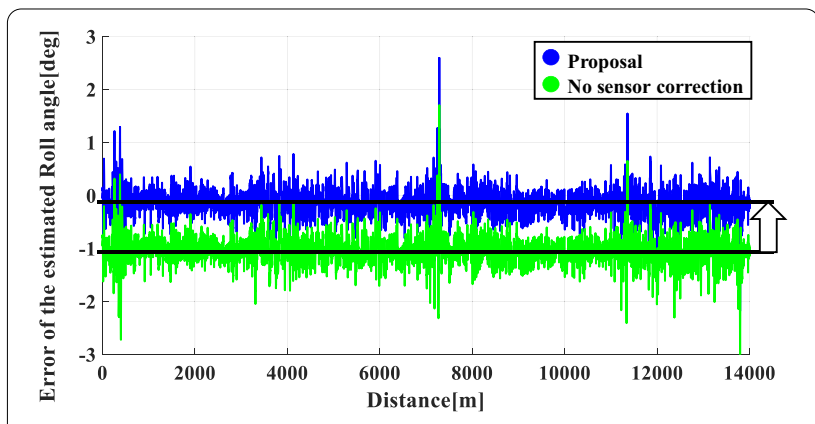

Fig. 13 Error of estimated roll angle (Urban highway)

Table 2 Error of estimated roll angle (Urban street)

\begin{tabular}{lll}
\hline Error & Average $\left(^{\circ}\right)$ & $\begin{array}{l}\text { Standard } \\
\text { deviation } \\
\left({ }^{\circ}\right)\end{array}$ \\
\hline Proposal & -0.09 & 0.22 \\
No sensor correction & -0.71 & 0.22 \\
\hline
\end{tabular}

Table 3 Error of estimated roll angle (Urban highway)

\begin{tabular}{lll}
\hline Error & Average $\left(^{\circ}\right)$ & $\begin{array}{l}\text { Standard } \\
\text { deviation } \\
\left({ }^{\circ}\right)\end{array}$ \\
\hline Proposal & -0.17 & 0.29 \\
No sensor correction & -1.06 & 0.29 \\
\hline
\end{tabular}

and 3 show the errors in the estimated roll angles. From Tables 2 and 3, it can be observed that the mean error was improved by the correction of the lateral acceleration error estimated by the proposed method for both the courses.

However, when comparing the two courses, urban street and highway, it can be confirmed that the average error is smaller for urban roads. The major differences between the two courses include the total distance traveled and the effect of multipath. Urban highway may be more susceptible to multipath effects due to the presence of high-rise buildings. In addition, the lateral acceleration error is estimated as a fixed value in this method. In the experiment on the urban expressway, the total driving distance is longer, and this bias error may have fluctuated slightly.

\section{Slip angle estimation results}

The slip angles estimated using the proposed method are shown in Figs. 14 and 15 for the urban street and highway, respectively. These figures prove that the estimated slip angles were close to the reference model for 


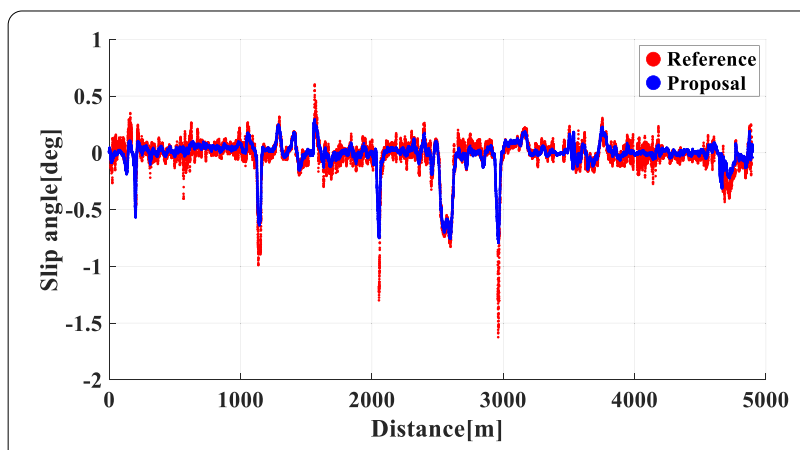

Fig. 14 Estimated slip angle (Urban street)

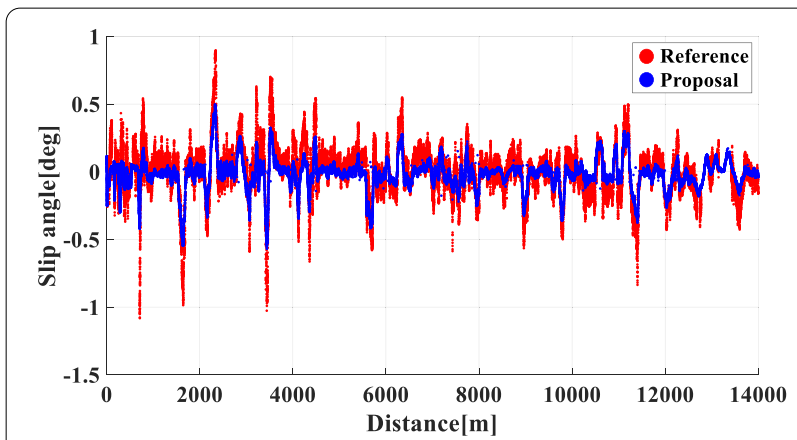

Fig. 15 Estimated slip angle (Urban highway)

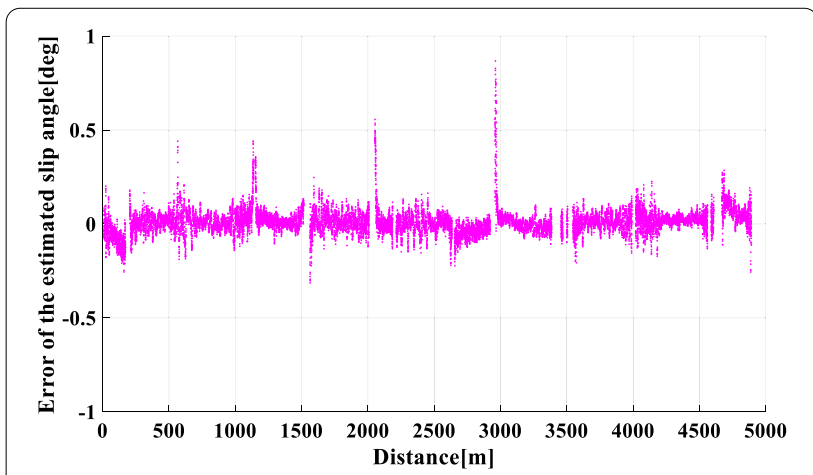

Fig. 16 Error of estimated slip angle (Urban street)

both the courses. Furthermore, the difference between the reference and estimated slip angles are presented in Figs. 16 and 17; the error in the estimated slip angle is shown in Table 4 . Table 4 shows that both courses were estimated with high accuracy, with a mean error of $0.01^{\circ}$. In these figures, it was also confirmed that there are some points where the error becomes slightly larger when focusing on the points (curves) where large slip angles occur. This is considered to be a point where the two-wheel model used in this method is not applicable.

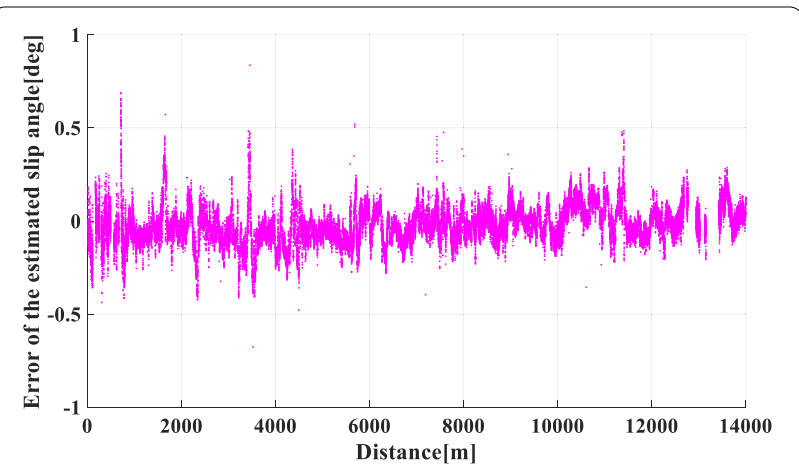

Fig. 17 Error of estimated slip angle (Urban highway)

Table 4 Error of estimated slip angle

\begin{tabular}{lcl}
\hline Error & Average $\left(^{\circ}\right)$ & $\begin{array}{l}\text { Standard } \\
\text { deviation } \\
\left({ }^{\circ}\right)\end{array}$ \\
\hline Urban street & 0.01 & 0.07 \\
Urban highway & -0.01 & 0.10 \\
\hline
\end{tabular}

\section{Trajectory estimation results}

Thereafter, the effect of the slip angle correction on the trajectory was evaluated. Figure 18 shows the results of using the corrected slip angle estimation for the trajectory obtained from the conventional method $[1,4]$ at the urban street shown in Fig. 8a. The trajectory before and after the slip angle correction is shown in the inset of Fig. 18, compared with the trajectory drawn from the starting point aligned to the reference. Figure 18 further shows that the trajectory beyond the turn of curve approached the reference model by correcting the slip angle, which improved the accuracy of the proposed model.

Since this study also aims to improve the accuracy of the trajectory estimation performance for normal driving, we will evaluate the estimation performance of the estimated vehicle trajectory. In this evaluation, we evaluate the error of a 100-m trajectory in order to understand the performance of the vehicle trajectory in a real environment. The number $100 \mathrm{~m}$ was chosen because vehicle trajectory-based vehicle control requires a distance of about $100 \mathrm{~m}$ when changing lanes. In this study, we evaluated the road orthogonal error of $100 \mathrm{~m}$ trajectory every $10 \mathrm{~m}$ on urban street and highway. The trajectory was set according to the wheel speed, IMU, and slip angle after aligning the POSLV220 only to the base point. Figure 19 shows an overview of the evaluation test; Figs. 20 and 21 show the results of the evaluation, where the accuracy of the trajectory was improved on both the urban street and the highway. In particular, 

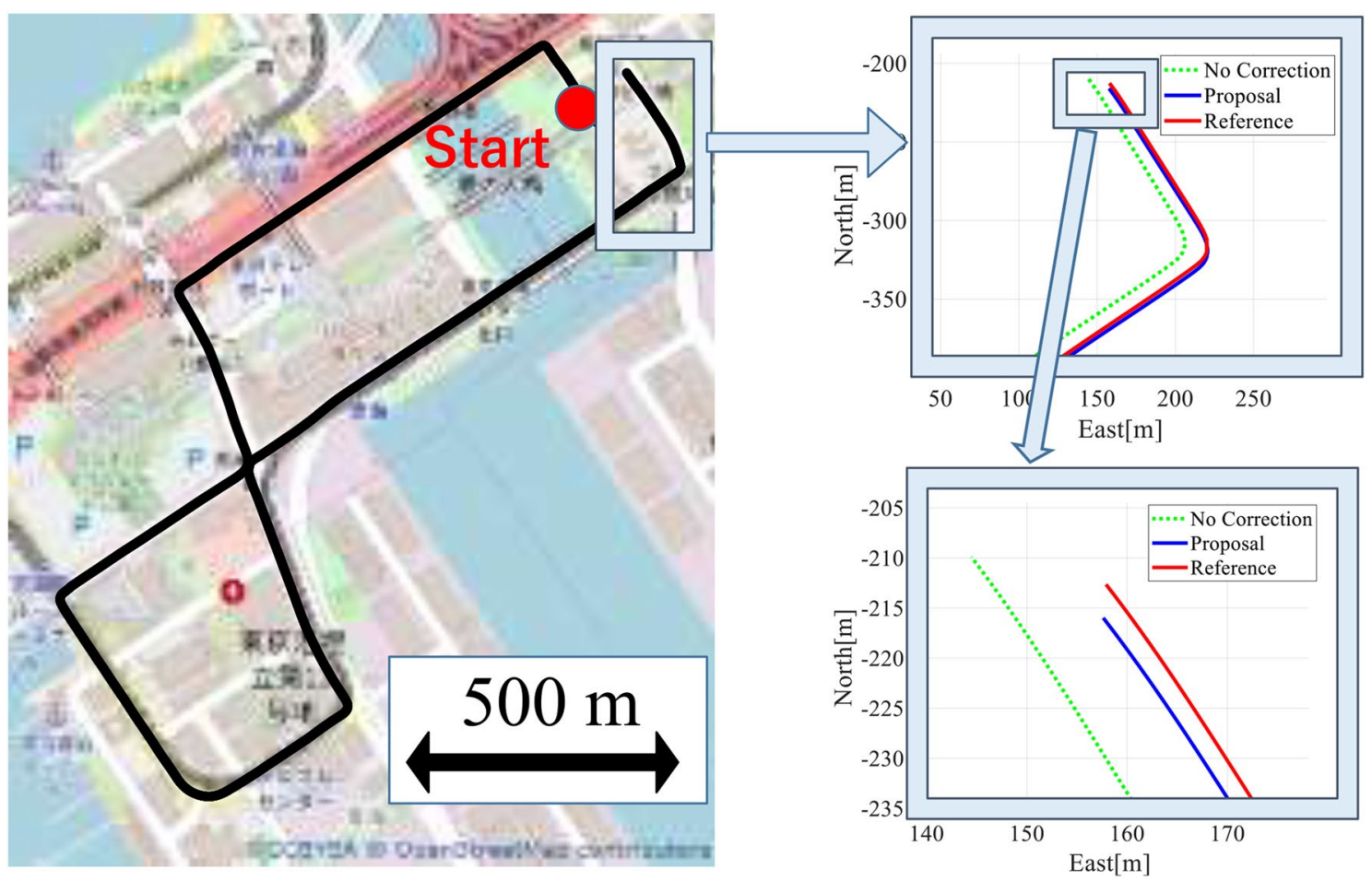

Fig. 18 Effect of slip angle correction (Urban street)

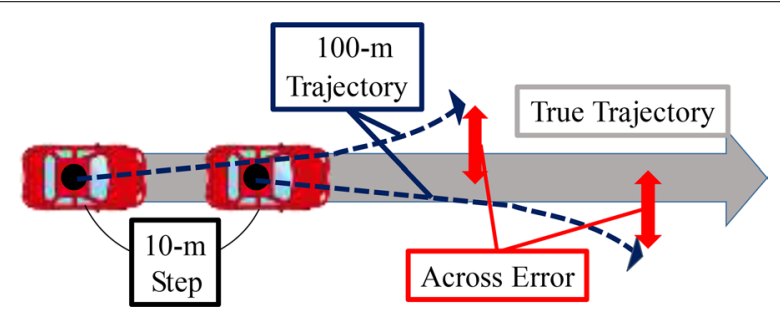

Fig. 19 Overview of trajectory evaluation

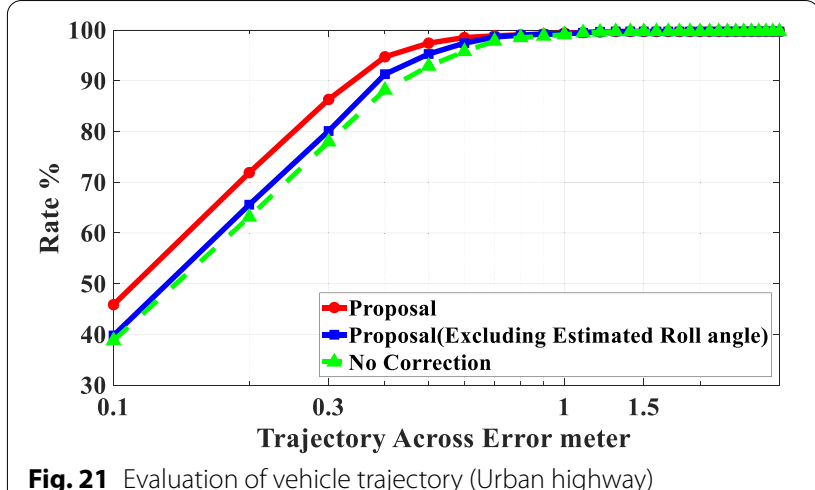

Fig. 21 Evaluation of vehicle trajectory (Urban highway)

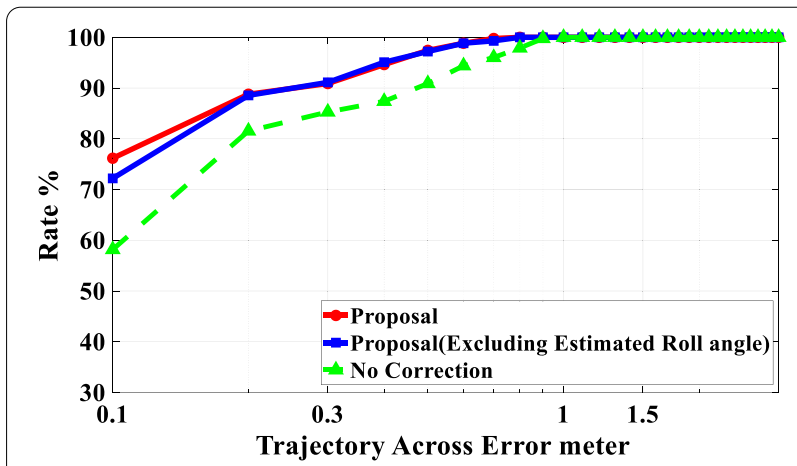

Fig. 20 Evaluation of vehicle trajectory (Urban street) the accuracy of the trajectory-at an error of $30 \mathrm{~cm}-$ was approximately $10 \%$ higher than that before the correction (no correction). Moreover, the estimation accuracy was improved by considering the roll angle in Eq. (14), as compared to excluding the roll angle $\phi$ in the given equation. The effect of considering the roll angle was particularly pronounced on the urban highway that has a large number of lateral slopes. 


\section{Conclusions}

In this paper, we focus on the estimation of slip angle to improve the accuracy of vehicle trajectory estimation, which is an important factor in automatic vehicle driving. In the conventional linear observer estimation method using a two-wheel model, which is a typical slip angle estimation method, the parameters of the two-wheel model are estimated one by one, and the bias of the error has been a problem.

Therefore, in this study, the two-wheel motorcycle model equation for the position of center of gravity was replaced by that for the center of the rear axle slip angle, and the parameters of the motorcycle model were collectively estimated as a gain $K$. This allowed the automatic estimation of parameters and ensured the removal of the steering angle term that was difficult to measure. We utilized the GNSS Doppler/IMU and GNSS Doppler to estimate the gain $K$. The difference between the heading angles measured by the GNSS Doppler/IMU and the velocity vectors measured by the GNSS Doppler from the motorcycle model was first-order approximated by the least-squares method, and the slope of the first-order equation was defined as the gain $K$. By accounting the effect of the roll angle for the estimation of the gain $K$, a highly accurate slip angle estimation was performed. The roll angle estimation was based on the relationship of the integrated yaw-rate value and the variation of the heading angle during a certain time period. This study enabled the estimation of the slip angle without the need for the observer and vehicle-specific tuning, and allowed the correction of slip to be easily incorporated into the trajectory estimation.

The effectiveness of the slip angle correction for the vehicular trajectory was confirmed in the evaluation test. The experiments were conducted on a standard urban (street) course and a high-speed (highway) course that was prone to lateral gradients; the error rates of the 100-m trajectory for both courses were improved by about $10 \%$ to cover within $30 \mathrm{~cm}$. The consideration of roll angle improved the accuracy of the estimation, and its effectiveness was especially significant on a course with numerous lateral slopes such as the urban highway.

In this study, an off-line processing was used to improve the accuracy of the trajectory estimation by estimating a highly accurate slip angle. This was effective for off-line use of trajectories such as the creation of 3-D maps for automated driving. As the theoretical capability of the proposed model has been verified for real-time use, we will continue to improve the algorithm for realtime implementation to further improve the usability of the proposed model.

\section{Acknowledgements}

Not applicable.

\section{Authors' contributions}

KT made a central contribution to the writing of the manuscript by proposing methods, conducting experiments, and analyzing data. All authors read and approved the final manuscript.

\section{Funding}

Not applicable.

\section{Availability of data and materials \\ Not applicable.}

\section{Competing interests}

The authors declare that they have no competing interests.

\section{Author details \\ ${ }^{1}$ Division of Mechatronics Engineering, Graduate School of Science and Tech- nology, Meijo University, Shiogamaguchi 1-501, Tenpaku-ku, Nagoya, Aichi 468-0073, Japan. ${ }^{2}$ Department of Mechatronics Engineering, Faculty of Sci- ence and Technology, Meijo University, Shiogamaguchi 1-501, Tenpaku-ku, Nagoya 468-0073, Aichi, Japan.}

Received: 8 October 2020 Accepted: 6 February 2021

Published online: 16 February 2021

\section{References}

1. Meguro J, Arakawa T, Mizutani S, Takanose A (2018) Low-cost lane-level positioning in urban area using optimized long time series GNSS and IMU Data. In: 2018 21st International Conference of Intelligent Transport Systems (IEEE ITSC), Maui, HI, pp 3097-3104, 2018. https://doi.org/10.1109/ itsc.2018.8569565

2. Ulbrich F, Rotter S, Goehring D, Rojas R (2016) Extracting path graphs from vehicle trajectories. In: 2016 IEEE Intelligent Vehicle Systems (IV), Gothenburg, Sweden, pp 1260-1264, 2016. https://doi.org/10.1109/ ivs.2016.7535552

3. Mukherjee S, Wang S, Wallace A (2020) Interacting vehicle trajectory prediction with convolutional recurrent neural networks. In: 2020 IEEE International Conference on Robotics and Automation (ICRA), Paris, France, pp 4336-4342, 2020. https://doi.org/10.1109/icra40945.2020.9196807

4. Kurzer K, Engelhorn F, Zollner JM (2018) Decentralized cooperative planning for automated vehicles with continuous Monte Carlo Tree Search. In: 2018 21st International Conference on Intelligent Transportation Systems (ITSC), Maui, HI, pp 452-459, 2018. https://doi.org/10.1109/ itsc.2018.8569988

5. Ljungqvist O, Axehill D, Pettersson H (2020) On sensing-aware model predictive path-following control for a reversing general 2-trailer with a car-like tractor. IEEE ICRA arXiv:2002.06874

6. Buckman N, Pierson A, Karaman S, Rus D (2020) Generating visibilityaware trajectories for cooperative and proactive motion planning. In: 2020 IEEE International Conference on Robotics and Automation (ICRA), pp 3220-3226, 2020. https://doi.org/10.1109/icra40945.2020.9196809

7. Jaipuria N, Habibi G, How JP (2018) Learning in the curbside coordinate frame for a transferable pedestrian trajectory prediction model. In: 2018 21st International Conference on Intelligent Transportation Systems (ITSC), Maui, HI, pp 3125-3131, 2018. https://doi.org/10.1109/ itsc.2018.8569812

8. Singh KB (2019) Vehicle sideslip angle estimation based on tire model adaptation. Electronics 8(2):199. https://doi.org/10.3390/electronics8020 199

9. Tanenhaus M, Geis T, Carhoun D, Holland A (2010) Accurate Real Time Inertial Navigation Device by Application and Processing of Arrays of MEMS Inertial Sensors. In: IEEE/ION Position, Location and Navigation Symposium, Indian Wells, CA, pp 20-26, 2010. https://doi.org/10.1109/ plans.2010.5507137

10. Perlmutter M, Robin L (2012) High-performance, low-cost intertial MEMS a market in motion! In: Proceedings of the 2012 IEEE/ION Position, Location and Navigation Symposium, Myrtle Beach, SC, pp 225-229, 2012. https://doi.org/10.1109/plans.2012.6236884

11. Takanose A, Atsumi Y, Takikawa K, Meguro J (2020) Height fluctuation kinematic positioning reliability, In: Proceedings of the 33rd International 
Technical Meeting of the Satellite Division of The Institute of Navigation (ION GNSS + 2020), pp $2710-2717$

12. Geng C, Mostefai L, Hori Y (2008) A Hybrid-like Observer of Body Slip Angle for Electric Vehicle Stability Control: Fuzzy Logic and Kalman Filter Approach. In: 2008 IEEE Vehicle Power and Propulsion Conference, Harbin, pp 1-6, 2008. https://doi.org/10.1109/vppc.2008.4677425

13. Ghosh J, Tonoli A, Amati N (2016) Sideslip angle estimation of a Formula SAE racing vehicle. SAE Int J Passeng Cars Mech Syst. https://doi. org/10.4271/2016-01-1662

14. Yoon JH, Peng H (2014) Robust vehicle sideslip angle estimation through a disturbance rejection filter that integrates a magnetometer with GPS. Intell Transp Syst IEEE Trans 15(1):191-204

15. Donald S, Corno M, Panzani G, Savaresi SM (2017) Vehicle sideslip estimation: a kinematic based approach. Control Eng. Pract. 67:1-12

16. Du H, Lam J, Cheung K-C, Li W, Zhang N (2015) Side-slip angle estimation and stability control for a vehicle with a non-linear tyre model and a varying speed. Proc Inst Mech Eng Part D 229:486-505

17. Pi DW, Chen N, Wang JX, Zhang BJ (2011) Design and evaluation of sideslip angle observer for vehicle stability control. Int J Automot Technol. 12:391-399

18. Iyabongkarn D, Rajesh R, John AG, Jae YL (2009) Development and experimental evaluation of a slip angle estimator for vehicle stability control. IEEE Trans Control Syst Technol 2009(17):78-88

19. Sierra C, Tseng E, Jain A, Peng H (2007) Cornering stiffness estimation based on vehicle lateral dynamics. Veh Syst Dyn. 44:24-38

20. Lundquist C, Schön TB (2009) Recursive identification of cornering stiffness parameters for an enhanced single track model. IFAC Proc Volumes 42:1726-1731

21. Viehweider A, Nam K, Fujimoto H, Hori Y (2012) Evaluation of a betaless instantaneous corneringstiffness estimation scheme for electric vehicles. In Proceedings of 2012 9th France-Japan \& 7th Europe-Asia Congress on Mechatronics (MECATRONICS)/13th Int'I Workshop on Research and Education in Mechatronics (REM), Paris, France, 21-23 November 2012

22. Gadola M, Chindamo D, Romano M, Padula F (2004) Development and Validation of a Kalman filter-based model for vehicle slip angle estimation. Veh Syst Dyn. 52(1):68-84
23. Rajamani R, Phanomchoeng G, Piyabongkarn D, Lew JY (2012) Algorithms for real-time estimation of individual wheel tire-road friction coefficients. IEEE/ASME Trans Mechatron 17(6):1183

24. Teerhuis A, Schmeitz A.J.C, Molengraft-Luijten, L (2015) Tire state estimation based on measured accelerations at the tire inner liner using an extended Kalman filter design. In Proceedings of the 4th International Tyre Colloquium, Tyre Models for Vehicle Dynamics Analysis, Ed. P. Gruber, RS Sharp

25. Sun R, Cheng Q (2020) Wang J (2020) Precise vehicle dynamic heading and pitch angle estimation using time-diferenced measurements from a single GNSS antenna. GPS Solut 24:84

26. Pereira R (2016) GPS Heading and Pitch Estimation using Single-Frequency, Dual-Frequency or Wide-Lane Measurements, IT - Instituto de Telecomunicac, o $\mathrm{O}^{2} \mathrm{es}$

27. Wang H, Liu N, Su Z, Li Q (2019) Research on low-cost attitude estimation for MINS/dual-antenna GNSS integrated navigation method. Micromachines 10(6):362

28. Tseng HE, Xu L, Hrovat D (2006) Estimation of Land Vehicle Roll and Pitch Angles. Veh Syst Dyn. 45(5):433. https://doi.org/10.1080/0042311060 1169713

29. Kojima Y (2010) Precise localization using tightly coupled integration based on trajectory estimated from GPS Doppler. Proceedings of AVEC 168:1-6

30. Takeyama K, Kojima Y, Teramot E (2012) Trajectory estimation improvement based on time-series constraint of GPS Doppler and INS in urban areas. Proc IEEE/ION PLANS 2012:700-705

31. POS-LV-Datasheet https://www.applanix.com/downloads/products/ specs/POS-LV-Datasheet.pdf. Accessed 17 September 2020

32. Serrano L, Kim D, Langley RB (2004) A GPS velocity sensor: How accurate can it be?-A first look. San Diego CA, pp.875-885, 2004

\section{Publisher's Note}

Springer Nature remains neutral with regard to jurisdictional claims in published maps and institutional affiliations.

\section{Submit your manuscript to a SpringerOpen ${ }^{\circ}$ journal and benefit from:}

- Convenient online submission

- Rigorous peer review

- Open access: articles freely available online

- High visibility within the field

- Retaining the copyright to your article

Submit your next manuscript at $\boldsymbol{\nabla}$ springeropen.com 